\title{
Global IRS-1 phosphorylation analysis in insulin resistance
}

\author{
P. Langlais $\cdot$ Z. Yi $\cdot$ J. Finlayson $\cdot$ M. Luo $\cdot$ R. Mapes $・$ \\ E. De Filippis • C. Meyer • E. Plummer • \\ P. Tongchinsub $\cdot$ M. Mattern $\cdot$ L. J. Mandarino
}

Received: 29 March 2011 /Accepted: 8 July 2011 / Published online: 18 August 2011

(C) Springer-Verlag 2011

\begin{abstract}
Aims/hypothesis IRS-1 serine phosphorylation is often elevated in insulin resistance models, but confirmation in vivo in humans is lacking. We therefore analysed IRS-1 phosphorylation in human muscle in vivo.

Methods We used HPLC-electrospray ionisation (ESI)MS/MS to quantify IRS-1 phosphorylation basally and after insulin infusion in vastus lateralis muscle from lean healthy, obese non-diabetic and type 2 diabetic volunteers. Results Basal Ser323 phosphorylation was increased in type 2 diabetic patients $(2.1 \pm 0.43, p \leq 0.05$, fold change vs lean controls). Thr495 phosphorylation was decreased in type 2 diabetic patients $(p \leq 0.05)$. Insulin increased IRS-1
\end{abstract}

Electronic supplementary material The online version of this article (doi:10.1007/s00125-011-2271-9) contains peer-reviewed but unedited supplementary material, which is available to authorised users.

P. Langlais $\cdot$ Z. Yi $\cdot$ J. Finlayson $\cdot$ M. Luo $\cdot$ R. Mapes $\cdot$

E. De Filippis $\cdot$ C. Meyer $\cdot$ P. Tongchinsub $\cdot$ M. Mattern $\cdot$

L. J. Mandarino $(\square)$

Center for Metabolic and Vascular Biology,

School of Life Science, Arizona State University,

ISTB1, 550 E. Orange St,

Tempe, AZ 85287, USA

e-mail: lawrence.mandarino@asu.edu

Z. Yi

Department of Pharmaceutical Sciences, Eugene Applebaum

College of Pharmacy/Health Sciences, Wayne State University,

Detroit, MI, USA

C. Meyer $\cdot$ E. Plummer

Division of Endocrinology, Carl T. Hayden VA Medical Center,

Mayo Clinic in Arizona,

Phoenix, AZ, USA

L. J. Mandarino

Department of Medicine, Mayo Clinic in Arizona,

Scottsdale, AZ, USA phosphorylation at Ser527 (1.4 $\pm 0.17, p \leq 0.01$, fold change, $60 \mathrm{~min}$ after insulin infusion vs basal) and Ser531 (1.3 \pm 0.16 , $p \leq 0.01$, fold change, $60 \mathrm{~min}$ after insulin infusion vs basal) in the lean controls and suppressed phosphorylation at Ser348 $(0.56 \pm 0.11, p \leq 0.01$, fold change, $240 \mathrm{~min}$ after insulin infusion vs basal), Thr446 (0.64 $\pm 0.16, p \leq 0.05$, fold change, $60 \mathrm{~min}$ after insulin infusion vs basal), Ser1100 $(0.77 \pm 0.22, p \leq 0.05$, fold change, $240 \mathrm{~min}$ after insulin infusion vs basal) and $\operatorname{Ser} 1142(1.3 \pm 0.2, p \leq 0.05$, fold change, $60 \mathrm{~min}$ after insulin infusion vs basal).

Conclusions/interpretation We conclude that, unlike some aspects of insulin signalling, the ability of insulin to increase or suppress certain IRS-1 phosphorylation sites is intact in insulin resistance. However, some IRS-1 phosphorylation sites do not respond to insulin, whereas other Ser/Thr phosphorylation sites are either increased or decreased in insulin resistance.

Keywords Insulin resistance $\cdot$ IRS-1 1 Mass spectrometry Serine $\cdot$ Phosphorylation $\cdot$ Threonine $\cdot$ Type 2 diabetes

$\begin{array}{ll}\text { Abbreviations } \\ \text { CID } & \text { Collision-induced dissociation } \\ \text { ESI } & \text { Electrospray ionisation } \\ \text { IR } & \text { Insulin receptor } \\ \text { LTQ-FTICR } & \begin{array}{l}\text { Linear trap quadrupole/Fourier transform } \\ \text { ion cyclotron resonance }\end{array} \\ \text { PI3-K } & \text { Phosphatidylinositol 3-kinase } \\ \text { PTB } & \text { Phosphotyrosine binding }\end{array}$

\section{Introduction}

Insulin-stimulated tyrosine phosphorylation of IRS-1 by the insulin receptor (IR) results in intracellular transduction of the insulin signal, whereas serine/threonine phosphorylation 
modulates the function of tyrosine-phosphorylated IRS-1 in either a positive or negative manner [1]. Phosphorylation of IRS-1 is increased at select serine residues in models of insulin resistance and type 2 diabetes [2]. Elevated Ser/Thr IRS-1 phosphorylation may inhibit insulin signalling by (1) downregulating IRS-1 protein levels through ubiquitination [3-7], (2) inhibiting the interaction between the tyrosinephosphorylated IR and the phosphotyrosine binding (PTB) domain of IRS-1 [8-14], or (3) interfering with the insulinstimulated interaction between IRS-1 and its downstream partner, the phosphatidylinositol 3-kinase (PI3-K) adaptor molecule $\mathrm{p} 85[10,15,16]$.

Although insulin stimulates an increase in Tyr phosphorylation of IRS-1, Ser/Thr phosphorylation of IRS-1 can be increased, suppressed or remain unchanged upon insulin stimulation. Ser/Thr phosphorylation of IRS-1 can positively regulate IRS-1 [17], although the majority of IRS-1 Ser/Thr phosphorylation has generally been regarded as a negative regulator of IRS-1 function [1]. Paradoxically, many of the proposed inhibitory human IRS-1 phosphorylation sites, such as Ser312 (equivalent to murine/rat Ser307), Ser616 (murine/rat Ser612) and Ser636 (murine/rat Ser632) can undergo acute insulin stimulation simultaneously with IRS-1 Tyr phosphorylation $[18,19]$. Recently, Ser307Ala knock-in mice were found to be insulin resistant [20], suggesting that Ser307 phosphorylation may be important for insulinstimulated IRS-1 function, despite previous reports that phosphorylation at this site inhibits IRS-1 association with the IR [21]. These complex and seemingly contradictory findings provide a compelling rationale for a more comprehensive understanding of IRS-1 phosphorylation in insulin resistance in vivo in humans.

To study IRS-1 Ser/Thr phosphorylation in vivo in human muscle, we devised a MS approach to simultaneously quantify the effect of insulin on multiple IRS-1 phosphorylation sites $[22,23]$. In the current study, we have assessed differences in IRS-1 phosphorylation in vastus lateralis muscle among lean healthy, obese non-diabetic and type 2 diabetic human participants basally and during a euglycaemic, hyperinsulinaemic clamp. To assess the time course of the ability of insulin to change IRS-1 phosphorylation, we performed biopsies at $60 \mathrm{~min}$ of insulin infusion to determine the acute, more physiological effects, and after $4 \mathrm{~h}$ of insulin in order to determine the more chronic effects of hyperinsulinaemia.

\section{Methods}

Materials The following suppliers were used: sequencinggrade trypsin and protein A sepharose (Sigma, St Louis, MO, USA); C18 ZipTip (Millipore, Billerica, MA, USA); antibody to IRS-1 (Upstate/Millipore, Billerica, MA, USA).
Participants Eight lean, healthy individuals without a family history of type 2 diabetes, eight obese insulinresistant individuals with normal glucose tolerance, and eight individuals with type 2 diabetes took part in the study. None of the participants had any significant medical problems (other than diabetes), and their weight had been stable for at least 3 months before the study. None engaged in any heavy exercise, and they were instructed not to engage in vigorous exercise for at least 3 days before the study. Patients with diabetes were treated by diet alone, sulfonylureas or insulin. Sulfonylureas were withdrawn 2 days before, and insulin was withdrawn the night before the study. The purpose, nature and potential risks of the study were explained to all participants, and written consent was obtained before their participation. The protocol was approved by the institutional review boards of Arizona State University and the Carl T. Hayden VA Medical Center. All participants received a $75 \mathrm{~g}$ oral glucose tolerance test on a separate day to assess glucose tolerance.

Euglycaemic clamp with muscle biopsies A euglycaemic, hyperinsulinaemic clamp was used to assess insulin sensitivity and expose skeletal muscle to insulin in vivo, as previously described $[22,24,25]$. On the day of study, participants reported to the Clinical Research Unit at Arizona State University or Carl T. Hayden VA Medical Center following an overnight fast. At 07:00 hours (-120 min), a catheter was placed in an antecubital vein and maintained throughout the study for infusions of insulin and glucose. A second catheter was placed in a retrograde manner into a vein on the back of the hand, which was then placed in a heated box $\left(60^{\circ} \mathrm{C}\right)$. Baseline arterialised venous blood samples for determination of plasma glucose and insulin concentrations were drawn. At 08:00 hours (time $-60 \mathrm{~min}$ ), after a $60 \mathrm{~min}$ rest, a percutaneous needle biopsy of the vastus lateralis muscle was performed under local anaesthesia [24]. After a tracer $\left(\left[6,6^{2} \mathrm{H}\right]\right.$ glucose $)$ equilibration period $(0.04 \mathrm{mg} \mathrm{kg} / \mathrm{min}$, $120 \mathrm{~min})$, at 09:00 hours (0 $\mathrm{min})$, a primed, continuous infusion of human regular insulin (Novolin; Novo Nordisk Pharmaceuticals, Princeton, NJ, USA) was started at a rate of $80 \mathrm{mU} \mathrm{m}^{-2} \mathrm{~min}^{-1}$ and continued for $240 \mathrm{~min}$. Arterialised blood samples were collected every $5 \mathrm{~min}$ for plasma glucose determination, and a $20 \%$ glucose infusion was adjusted to maintain the plasma glucose concentration at each participant's fasting plasma glucose level. Glucose turnover rates were determined using either $\left[6-{ }^{3} \mathrm{H}\right]$ glucose or $\left[6,6-{ }^{2} \mathrm{H}\right]$ glucose. For the $\left[6,6-^{2} \mathrm{H}\right]$ glucose method, the $20 \%$ dextrose solution was enriched with $\left[6,6-{ }^{2} \mathrm{H}\right]$ glucose, and the constant infusion of $\left[6,6-^{2} \mathrm{H}\right]$ glucose was stopped $+30 \mathrm{~min}$ into the clamp. Tracer to tracee ratio of glucose $\left(\left[6,6{ }^{2} \mathrm{H}\right]\right.$ glucose/glucose) was determined via the derivatisation product, glucose penta-acetate, using the Finnigan Trace DSQ GS/MS (Thermo Electron Corporation, Waltham, MA, USA). 
Selective ion monitoring was used to determine glucose tracer enrichments using fragments ions with $\mathrm{m} / \mathrm{z} 200$ and 202 , as previously described [26]. $\left[6-{ }^{3} \mathrm{H}\right]$ Glucosebased determination of glucose appearance and disposal rates was performed as previously described [27]. Hepatic glucose production and glucose disposal were calculated using steady-state equations [28]. At time 10:00 hours (+60 min after the start of insulin infusion), a second percutaneous muscle biopsy sample was obtained from the contralateral vastus lateralis muscle, and a third percutaneous muscle biopsy sample was subsequently obtained at 13:00 hours ( $+240 \mathrm{~min}$ after the start of insulin infusion) distal to the primary vastus lateralis muscle biopsy site.

Analytical determinations Plasma glucose was measured by the glucose oxidase method (Beckman Instruments, Fullerton, CA, USA). Plasma insulin concentration was measured by ELISA (Alpco Diagnostics, Salem, NH, USA).

Muscle biopsy processing Approximately 60-130 mg frozen muscle biopsy samples were homogenised on ice using a Brinkman Homogenizer (Model PT 10/35) in detergentcontaining lysis buffer A $(50 \mathrm{mmol} / \mathrm{l}$ Hepes, $\mathrm{pH} 7.6$, $150 \mathrm{mmol} / \mathrm{l} \mathrm{NaCl}, 20 \mathrm{mmol} / 1 \mathrm{NaPO}_{4}, 20 \mathrm{mmol} / \mathrm{l}$ $\beta$-glycerophosphate, $10 \mathrm{mmol} / 1 \mathrm{NaF}, 2 \mathrm{mmol} / 1$ sodium vanadate, $2 \mathrm{mmol} / 1$ EDTA, $1 \%$ Triton, $10 \%$ glycerol, $2 \mathrm{mmol} / 1$ phenylmethylsulfonyl fluoride (PMSF), $1 \mathrm{mmol} / 1 \mathrm{MgCl}_{2}, 1 \mathrm{mmol} / 1 \mathrm{CaCl}_{2}, 10 \mu \mathrm{g} / \mathrm{ml}$ leupeptin, $10 \mu \mathrm{g} / \mathrm{ml}$ aprotinin) at a $100 \mu \mathrm{l} / 10 \mathrm{mg}$ ratio. Biopsy specimens were homogenised until no visible muscle remained $(\sim 3 \times 10 \mathrm{~s}$ pulses). Muscle lysates were then incubated on ice for $20 \mathrm{~min}$ followed by centrifugation at $4^{\circ} \mathrm{C}$ for $20 \mathrm{~min}$ at $14 \times$ $1,000 \mathrm{rpm}$. Protein concentrations in the supernatant fractions were determined by the method of Lowry et al. [29].

Immunoprecipitation, one-dimensional SDS-PAGE, and in-gel digestion IRS-1 was immunoprecipitated from $\sim 6-13 \mathrm{mg}$ muscle lysate (protein concentration averaged $\sim 10 \mu \mathrm{g} / \mu \mathrm{l}$ ) using $2 \mu \mathrm{g}$ antibody to IRS-1 (catalogue no. 06-248; Upstate/Millipore) coupled to $25 \mu$ l Protein A Sepharose beads (catalogue no. P3391; Sigma) overnight at $4^{\circ} \mathrm{C}$ with gentle rotation. The immunoprecipitates were spun down and washed four times in $1 \mathrm{ml}$ PBS solution. The remaining PBS solution was removed, the immunoprecipitates were boiled in $15 \mu \mathrm{l}$ sample loading buffer, and the proteins were resolved by $10 \%$ SDS-PAGE. The in-gel digestions were performed exactly as previously described [23].

Mass spectrometry High-performance liquid chromatography/electrospray ionisation/tandem mass spectrometry (HPLC-ESI-MS/MS) was performed on a Thermo Finnigan (San Jose, CA, USA) linear trap quadrupole/Fourier transform ion cyclotron resonance (LTQ-FTICR) fitted with a
PicoView nanospray source (New Objective, Woburn, MA, USA). On-line HPLC was performed using a Michrom BioResources Paradigm MS4 micro two-dimensional HPLC column (Alburn, CA, USA) with a PicoFrit column (New Objective; $75 \mu \mathrm{m}$ internal diameter; packed with ProteoPepTM II C18 material, $30 \mathrm{~nm}$; mobile phase, linear gradient of $2-27 \%$ acetonitrile $(\mathrm{ACN})$ in $0.1 \%$ formic acid in $65 \mathrm{~min}$, a hold of $5 \mathrm{~min}$ at $27 \% \mathrm{ACN}$, followed by a step to $50 \% \mathrm{ACN}$, hold $5 \mathrm{~min}$ and then a step to $80 \%$; flow rate, $400 \mathrm{nl} / \mathrm{min}$ ).

A 'top-10' data-dependent MS/MS analysis was performed (acquisition of a full scan spectrum followed by collision-induced dissociation (CID) mass spectra of the ten most abundant ions in the survey scan) to identify IRS-1 peptides and to obtain their HPLC retention times. For quantification, the following multisegment strategy was used: one survey scan, followed by one parent-list CID scan and six targeted CID scans. Included in the parent list were the $2+$ or $3+$ charge states of seven representative IRS-1 peptides selected from the prominent ions reproducibly observed in the top-10 data-dependent tandem-MS analysis. These peptides were used as internal standards for IRS-1 protein content (electronic supplementary material [ESM] Table 1). They were selected according to the following criteria: (1) detected by HPLC-ESI-MS with high intensity among IRS-1 peptides; (2) no missed cleavage observed; (3) no methionine in the sequence to avoid variability due to methionine oxidisation and no N-terminal Gln residues. The $2+$ or $3+$ ions of the phosphopeptides of interest were placed on the target list. In order to assess the relative quantities of a large number of phosphopeptides in each experiment and yet still maintain acceptable mass analysis cycle times, we grouped the targeted $\mathrm{m} / \mathrm{z}$ values into segments on the basis of their expected HPLC retention times with a minimum 10 min retention time window for each peptide.

Tandem mass spectra were extracted from Xcalibur 'RAW' files, and charge states were assigned using the Extract_MSN script that is a component of Xcalibur 2.0 SR2 (Thermo Fisher, San Jose, CA, USA). The fragment mass spectra were then searched against the human SwissProt_v52.2 database (16,135 entries) using Mascot (Matrix Science, London, UK; version 2.1). The search variables that were used were: $5 \mathrm{ppm}$ mass tolerance for precursor ion masses and $0.5 \mathrm{Da}$ for product ion masses; digestion with trypsin; a maximum of two missed tryptic cleavages; variable modifications of oxidation of methionine and phosphorylation of serine, threonine and tyrosine. Crosscorrelation of Mascot search results with X! Tandem was accomplished with Scaffold (version Scaffold-01_06_19; Proteome Software, Portland, OR, USA). Probability assessment of peptide assignments and protein identifications were made through the use of Scaffold. Only peptides with $\geq 95 \%$ 
probability were considered. Assignments of the phosphopeptides were confirmed by manual comparison of the tandem mass spectra with the predicted fragmentation generated in silico by the MS-Product component of Protein Prospector (http://prospector.ucsf.edu).

Peak areas for each peptide were obtained by integration of the appropriate reconstructed ion chromatograms with $5 \mathrm{ppm}$ error tolerance for precursor ion masses acquired using the FTICR and $0.5 \mathrm{D}$ for the fragment ions acquired using the LTQ mass analyser (see Table 2). The reconstructed ion chromatograms allow one to express the intensity (abundance) of ions relative to HPLC retention times in chromatogram form, analogously to analysis of UV absorbance during an HPLC run. The peak areas (using MS2/MS3 fragment ions) for the phosphopeptides were then normalised against the average peak area (using precursor ions) for the seven representative IRS-1 peptides (endogenous standards). Relative quantification of each phosphopeptide was obtained by comparing normalised peak/area ratios for control and insulin-treated samples.

Statistical analysis Statistical significance was assessed by comparing basal and insulin-stimulated phosphopeptide intensities (normalised to IRS-1 peptides as described above) using analysis of variance with post hoc $t$ tests. Differences were considered statistically significant at $p \leq 0.05$.

\section{Results}

Subject characteristics and insulin-stimulated glucose metabolism Three groups ( $n=8$ each) were studied; lean, healthy controls, obese non-diabetic volunteers, and participants with type 2 diabetes. Obese non-diabetic and type 2 diabetic participants had a higher BMI than lean control volunteers (Table 1). As expected, diabetic and obese nondiabetic participants were insulin resistant compared with lean, non-diabetic controls $(p<0.05)$. Quantification of
IRS-1 protein, based on the average peak area of the IRS-1 standard peptides, revealed no significant change of IRS-1 protein abundance in obese and type 2 diabetes muscle compared with the lean control (ESM Table 3).

IRS-1 serine and threonine phosphorylation analysis in human vastus lateralis muscle Participants underwent a $4 \mathrm{~h}$ euglycaemic, hyperinsulinaemic clamp as described in the Methods section, and biopsy specimens from vastus lateralis muscle were taken $1 \mathrm{~h}$ before insulin infusion (basal) and after both 1 and $4 \mathrm{~h}$ of insulin infusion. Tryptic digests of IRS-1 immunoprecipitated from muscle homogenates were analysed by targeted HPLC-ESI-MS to quantify IRS-1 phosphorylation under the various conditions, as previously described [23].

We assigned 24 phosphorylation sites in IRS-1 from human muscle; 16 were quantifiable by the MS2/MS3based MS technique (Table 2). Ser303, Ser323, Ser527, Ser531, Ser1142 and Ser1145 are representative of the IRS-1 phosphorylation sites that were newly quantifiable as a result of using the MS2/MS3 fragment ion approach. Our previous study on IRS-1 phosphorylation from muscle could not quantify these phosphorylation sites [22], because the different parent ions that share a phosphorylation site coelute as a mixture of singly phosphorylated peptides of the same mass ('isobaric'), rendering them indistinguishable during FTICR detection. Eight phosphorylation sites could not be quantified because of ambiguous peak areas (Ser892 and Ser1223), low abundance (Ser574 and Ser616), poor fragmentation (Ser1222) or uncertain residue identification relative to other phosphorylation sites within the same peptide (Ser307, Ser312 and Ser1143).

Effect of insulin on IRS-1 phosphorylation in lean, insulin-sensitive human muscle Of the 16 IRS-1 phosphorylation sites quantified in lean controls, six responded significantly to insulin treatment at either the +60 or $+240 \mathrm{~min}$ time points (either increased or decreased): Ser348 $(0.6 \pm 0.1$ vs basal, $p \leq 0.01 ;+240 \mathrm{~min})$, Thr446 $(0.6 \pm 0.2$,
Table 1 Participant characteristics

Data are given as means \pm SEM

Glucose disposal rates were calculated as the average value during the final $30 \mathrm{~min}$ of insulin infusion

${ }^{*} p \leq 0.05, * * p \leq 0.01, * * * p \leq 0.001$ (ANOVA)

T2D, type 2 diabetes

\begin{tabular}{lccc}
\hline Characteristic & Lean & Obese & T2D \\
\hline Age (years) & $39 \pm 4$ & $46 \pm 4$ & $48 \pm 3$ \\
Sex (male/female) & $4 / 4$ & $4 / 4$ & $3 / 5$ \\
BMI (kg/m²) & $24 \pm 0.9$ & $28 \pm 1^{*}$ & $34 \pm 2^{* * *}$ \\
Weight (kg) & $74 \pm 5$ & $81 \pm 5$ & $98 \pm 5^{* * *}$ \\
Fasting glucose (mmol/l) & $5.22 \pm 0.09$ & $5.46 \pm 0.14$ & $6.83 \pm 0.43^{* *}$ \\
Fasting insulin (pmol/l) & $41 \pm 9$ & $121 \pm 23^{* *}$ & $79 \pm 18$ \\
Basal glucose production (mg m & $\left.\mathrm{min}^{-1}\right)$ & $74.2 \pm 6.3$ & $80.3 \pm 4.0$ \\
Insulin-stimulated glucose disposal $\left(\mathrm{mg} \mathrm{m}^{-2} \mathrm{~min}^{-1}\right)$ & $465.3 \pm 90.1$ & $282.5 \pm 20.8^{*}$ & $233.4 \pm 23.2^{* *}$ \\
\hline
\end{tabular}


Table 2 IRS-1 fragment ions for quantification

\begin{tabular}{|c|c|c|c|c|c|c|}
\hline Start & Stop & Peptide sequence & Phosphorylation site & MS2 or MS3? & $\begin{array}{l}\text { Product ion } \\
\text { (charge state) }\end{array}$ & Mass \\
\hline \multirow[t]{5}{*}{303} & 325 & SRTESITATSPASMVGGKPGSFR & Ser303 & MS3 & y21 (+2) & $1,049.02$ \\
\hline & & & Ser307 & $\mathrm{x}$ & $\mathrm{x}$ & $\mathrm{x}$ \\
\hline & & & Ser312 & $\mathrm{x}$ & $\mathrm{x}$ & $\mathrm{x}$ \\
\hline & & & Ser323 & MS3 & b14 $(+1)$ & $1,436.67$ \\
\hline & & & & & y9 $(+1)$ & 886.49 \\
\hline \multirow[t]{6}{*}{328} & 353 & ASSDGEGTMSRPASVDGSPVSPSTNR & Ser330 & MS2 & y20 (+2) & $1,009.98$ \\
\hline & & & & MS2 & y22 (+2) & $1,103.01$ \\
\hline & & & & MS2 & y11 $(+1)$ & $1,116.53$ \\
\hline & & & Ser348 & MS2 & y8 $(+1)$ & 937.41 \\
\hline & & & & MS2 & y20 (+2) & $1,049.96$ \\
\hline & & & & MS2 & b11 $(+1)$ & $1,095.44$ \\
\hline \multirow[t]{3}{*}{444} & 457 & SVTPDSLGHTPPAR & Thr446 & MS3 & y9 $(+1)$ & 935.51 \\
\hline & & & & MS3 & y10 (+1) & $1,050.53$ \\
\hline & & & & MS3 & y11 (+1) & $1,147.59$ \\
\hline \multirow[t]{3}{*}{494} & 520 & CTPGTGLGTSPALAGDEAASAADLDNR & Thr495 & MS2 & y14 (+1) & $1,375.61$ \\
\hline & & & & & y17 (+1) & $1,656.78$ \\
\hline & & & & & y18 (+1) & $1,743.81$ \\
\hline \multirow[t]{2}{*}{525} & 538 & THSAGTSPTITHQK & Ser527 & MS3 & y11 (+1) & $1,140.60$ \\
\hline & & & Ser531 & MS3 & y11 (+1) & $1,122.59$ \\
\hline 573 & 580 & HSAFVPTR & Ser574 & $\mathrm{x}$ & $\mathrm{x}$ & $\mathrm{x}$ \\
\hline 596 & 626 & GGHHRPDSSTLHTDDGYMPMSPGVAPVPSGR & Ser616 & $\mathrm{x}$ & $\mathrm{x}$ & $\mathrm{x}$ \\
\hline \multirow[t]{2}{*}{627} & 638 & KGSGDYMPMSPK & Ser629 & MS3 & {$[\mathrm{M}+2 \mathrm{H}-98-64]^{2+}$} & $624.29^{\mathrm{a}}$ \\
\hline & & & Ser636 & MS3 & {$[\mathrm{M}+2 \mathrm{H}-98-64]^{2+}$} & $624.29^{a}$ \\
\hline 892 & 922 & SPGEYVNIEFGSDQSGYLSGPVAFHSSPSVR & Ser892 & $\mathrm{x}$ & $\mathrm{x}$ & $\mathrm{x}$ \\
\hline 999 & 1016 & QSYVDTSPAAPVSYADMR & Ser1005 & MS2 & y11 (+1) & $1,193.56$ \\
\hline \multirow[t]{2}{*}{1075} & 1081 & VNLSPNR & Ser1078 & MS3 & y5 $(+1)$ & 568.32 \\
\hline & & & & & y6 $(+1)$ & 682.36 \\
\hline 1098 & 1112 & RHSSETFSSTPSATR & Ser1100 & MS3 & y12 (+1) & $1,270.59$ \\
\hline \multirow[t]{2}{*}{1099} & 1112 & HSSETFSSTPSATR & Ser1101 & MS3 & b8 $(+1)$ & 845.34 \\
\hline & & & & & y10 (+1) & $1,054.52$ \\
\hline \multirow[t]{7}{*}{1141} & 1161 & HSSASFENVWLRPGELGGAPK & Ser1142 & MS3 & y11 (+1) & $1,183.559$ \\
\hline & & & & & y19 (+2) & $1,008.02$ \\
\hline & & & & & y18 (+2) & 964.50 \\
\hline & & & & & y17 (+2) & 928.99 \\
\hline & & & Ser1143 & $\mathrm{x}$ & $\mathrm{x}$ & $\mathrm{x}$ \\
\hline & & & Ser1145 & MS3 & y19 (+2) & 999.02 \\
\hline & & & & & y18 (+2) & 955.50 \\
\hline \multirow[t]{3}{*}{1221} & 1236 & RSSEDLSAYASISFQK & Ser1222 & $\mathrm{x}$ & $\mathrm{x}$ & $\mathrm{x}$ \\
\hline & & & & & $\mathrm{x}$ & $\mathrm{x}$ \\
\hline & & & Ser1223 & $\mathrm{x}$ & $\mathrm{x}$ & $\mathrm{x}$ \\
\hline
\end{tabular}

${ }^{a}$ The highest fragment ion in the MS3 spectrum, loss of phosphoric acid (98 Da) and $\mathrm{SOCH}_{4}(64 \mathrm{Da})$ from the parent ion. Peptides containing pSer629 and pSer636 were resolved by HPLC

$p \leq 0.05 ;+60 \mathrm{~min}), \operatorname{Ser} 527(1.4 \pm 0.2, p \leq 0.01 ;+60 \mathrm{~min})$, Ser531 ( $1.3 \pm 0.2, p \leq 0.01 ;+60 \mathrm{~min}), \operatorname{Ser} 1100 \quad(0.8 \pm 0.2$, $p \leq 0.05 ;+240 \mathrm{~min}), \operatorname{Ser} 1142(1.3 \pm 0.2, p \leq 0.05 ;+60 \mathrm{~min})$ (Table 3). The remaining ten IRS-1 phosphorylation sites showed no significant response to insulin (Ser303, Ser 323, Ser330, Thr495, Ser629, Ser636, Ser1005, Ser1078, Ser1101 and Ser1145). With regard to those sites that were significantly altered in phosphorylation by insulin infusion, there 
Table 3 IRS-1 phosphorylation in lean controls after 60 and 240 min of insulin infusion

\begin{tabular}{lll}
\hline $\begin{array}{l}\text { Phosphorylation } \\
\text { site }\end{array}$ & $\begin{array}{l}+60 \\
\text { Insulin }\end{array}$ & $\begin{array}{l}+240 \\
\text { Insulin }\end{array}$ \\
\hline Ser303 & $1.1 \pm 0.3$ & $0.7 \pm 0.2$ \\
Ser323 & $0.9 \pm 0.3$ & $0.9 \pm 0.2$ \\
Ser330 & $1.1 \pm 0.3$ & $1.1 \pm 0.3$ \\
Ser348 & $0.6 \pm 0.2$ & $0.6 \pm 0.1^{* *}$ \\
Thr446 & $0.6 \pm 0.2^{*}$ & $0.8 \pm 0.1$ \\
Thr495 & $0.8 \pm 0.2$ & $1.1 \pm 0.2$ \\
Ser527 & $1.4 \pm 0.2^{* *}$ & $1.5 \pm 0.3$ \\
Ser531 & $1.3 \pm 0.2^{* *}$ & $1.1 \pm 0.2$ \\
Ser629 & $0.7 \pm 0.2$ & $1.0 \pm 0.2$ \\
Ser636 & $1.3 \pm 0.6$ & $0.9 \pm 0.3$ \\
Ser1005 & $0.8 \pm 0.2$ & $0.9 \pm 0.2$ \\
Ser1078 & $0.9 \pm 0.2$ & $1.0 \pm 0.3$ \\
Ser1100 & $0.9 \pm 0.2$ & $0.8 \pm 0.2^{*}$ \\
Ser1101 & $1.3 \pm 0.3$ & $1.1 \pm 0.2$ \\
Ser1142 & $1.3 \pm 0.2^{*}$ & $1.0 \pm 0.3$ \\
Ser1145 & $1.0 \pm 0.2$ & $1.0 \pm 0.2$ \\
\hline
\end{tabular}

Effect of insulin over the $-60 \mathrm{~min}$ time point within group ( $t$ test): ${ }^{*} p \leq 0.05, * * p \leq 0.01$

were three patterns: (1) acutely increased at $+60 \mathrm{~min}$ (Ser527, Ser531 and Ser1142); (2) acutely suppressed at $+60 \mathrm{~min}$ (Thr446); and (3) gradually suppressed upon +240 min of insulin infusion (Ser348 and Ser1100).

\section{Elevated serine and threonine phosphorylation of IRS-1 in} type 2 diabetic muscle Of the IRS-1 phosphorylation sites analysed in this study, Ser323 possessed significantly increased levels of phosphorylation in the type 2 diabetic muscle compared with the lean controls (Fig. 1). Both Ser303 and Ser323 were hyperphosphorylated at every time point compared with the lean controls, although only Ser323 exhibited a significant increase $(2.1 \pm 0.43, p \leq 0.05$, fold change vs basal lean controls) (Fig. 1b).

Hypophosphorylation of IRS-1 in obese and type 2 diabetic muscle In our previous study analysing IRS-1 phosphorylation in human muscle, Thr495 was discovered as a new, previously unidentified phosphorylation site. Further analysis of this site in this study led to the finding that phosphorylation of Thr495 was significantly suppressed in both obese (basal: $0.32 \pm 0.13, p \leq 0.05 ; 60 \mathrm{~min}: 0.26 \pm 0.07$, $p \leq 0.05$; 240 min: $0.31 \pm 0.08, p \leq 0.01)$ and type 2 diabetic muscle (60 min: $0.36 \pm 0.05, p \leq 0.05 ; 240 \mathrm{~min}: 0.52 \pm 0.11$, $p \leq 0.05$ ) compared with the lean controls, at all time points studied, with the exception of the type 2 diabetic basal (Fig. 2b), revealing a potentially new mode of insulin resistance at the level of IRS-1 Ser/Thr phosphorylation.
Ser330 also exhibited significant hypophosphorylation in obese muscle (basal: $0.44 \pm 0.12, p \leq 0.05 ; 240 \mathrm{~min}: 0.37 \pm$ $0.14, p \leq 0.05)$, although unlike Thr495 phosphorylation, Ser330 phosphorylation levels were similar in lean and type 2 diabetic muscle (Fig. 2a).

Insulin effect on serine/threonine phosphorylation of IRS-1 in type 2 diabetic muscle Ser531 and Ser1142 of IRS-1 were found to undergo significant insulin stimulation in the lean participants, while Ser1100 phosphorylation was significantly suppressed (data and significance levels are shown in Table 3). Quantification of Ser531, Ser1100 and Ser1142 phosphorylation revealed that these IRS-1 phosphorylation sites did not significantly respond to insulin in the type 2 diabetic muscle (Fig. 3) relative to the lean

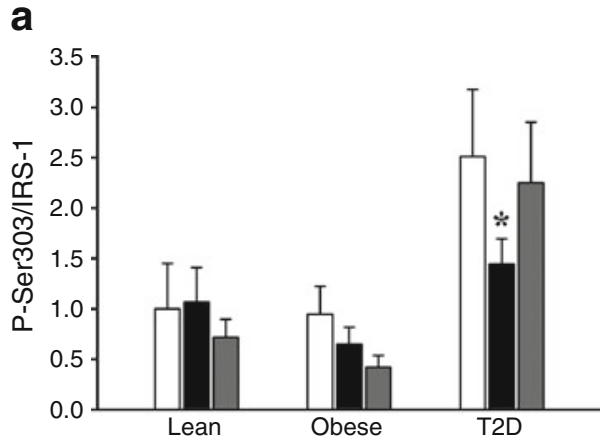

b

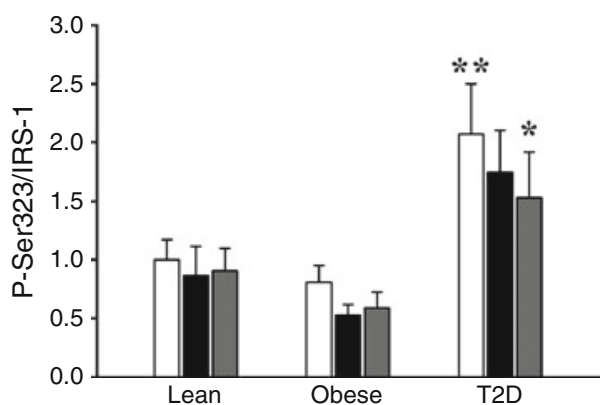

Fig. 1 Hyperphosphorylation of IRS-1 in type 2 diabetic human muscle in vivo. Lean controls, obese insulin sensitive controls, or type 2 diabetic human participants underwent a euglycaemic, hyperinsulinaemic (80 $\mathrm{mU} \mathrm{min}^{-1} \mathrm{~m}^{-2}$ body surface area) clamp, and vastus lateralis muscle biopsies were performed once basally ( $-60 \mathrm{~min}$, white bar) and twice after insulin infusion ( $+60 \mathrm{~min}$, black bar, and $+240 \mathrm{~min}$, grey bar). Tryptic digests of IRS-1 immunoprecipitated from muscle homogenates were then analysed by targeted HPLC-ESI-MS in order to quantify IRS-1 phosphorylation (as described in the Methods section). The peak areas for the phosphopeptides were normalised against the average peak area (using precursor ions) for the seven representative IRS-1 peptides. Each IRS-1 phosphorylation site was normalised by the mean value of the respective basal sample and then expressed as a fold change over basal \pm SEM $\left({ }^{*} p \leq 0.05\right.$; effect of insulin compared with the -60 min time point, within group; $t$ test. ${ }^{*} p \leq 0.05$ compared with the same time against lean, between groups; ANOVA). T2D, type 2 diabetes 

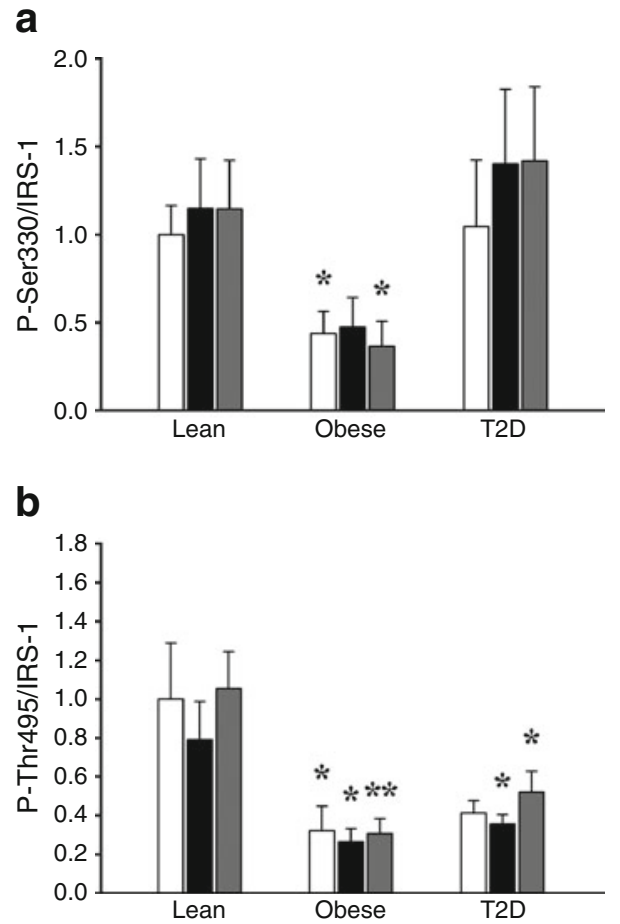

Fig. 2 Hypophosphorylation of IRS-1 in type 2 diabetic muscle in vivo. Experiments were performed identically to those described in Fig. $1 .{ }^{*} p \leq 0.05 ;{ }^{*} p \leq 0.01$ compared with the same time against lean, between groups; ANOVA. T2D, type 2 diabetes

controls, suggesting that, in addition to hyper-serine and hypo-threonine phosphorylation, IRS-1 serine/threonine phosphorylation can also fail to respond to insulin regulation or have a delayed response to insulin in the insulin-resistant state. Paradoxically, Ser348, Thr446 and Ser527, the other IRS-1 phosphorylation sites that were found to undergo significant insulin-induced changes in the lean controls, were not insulin resistant in the type 2 diabetic muscle, but rather, responded to insulin in the type 2 diabetic participants in a manner similar to that observed in the lean controls (Fig. 4). The remainder of the IRS-1 phosphorylation sites studied (Ser629, Ser636, Ser1005, Ser1078, Ser1101 and Ser1145) were either unaffected by insulin treatment or not significantly different between the different groups (Fig. 5). Quantification data for all IRS-1 phosphorylation sites can be found in ESM Table 2.

\section{Discussion}

We set out to test whether the IRS-1 phosphorylation sites that can be quantified by our MS-based approach exhibit altered levels of phosphorylation, basally or during insulin infusion, in vastus lateralis muscle from type 2 diabetic participants compared with lean healthy and obese nondiabetic controls (see Fig. 6 for a summary of the study). The lean controls had significant insulin-induced increases (Ser527, Ser531 and Ser1142) and decreases (Ser348, Ser446 and Ser1100) in IRS-1 phosphorylation. As for the type 2 diabetic muscle, Ser323 (murine/rat Ser318) was the prominent, hyperphosphorylated site located near the N-terminal PTB domain of IRS-1, potentially phosphorylated by kinases (p70S6K, protein kinase $\mathrm{C}[\mathrm{PKC}] \zeta$, c-Jun $\mathrm{N}$-terminal kinase [JNK], $\mathrm{PKC} \theta$, inhibitor $\mathrm{\kappa B}$ kinase $[\mathrm{IKK} \beta]$ ) that can be activated by inducers of insulin resistance such as elevated NEFA and cytokines, or by hyperglycaemia [30-41]. Ser323 phosphorylation has been proposed to play a role in the IRS-1 Ser/Thr phosphorylation negative feedback system [41-43] in which phosphorylation of specific IRS-1 Ser/Thr

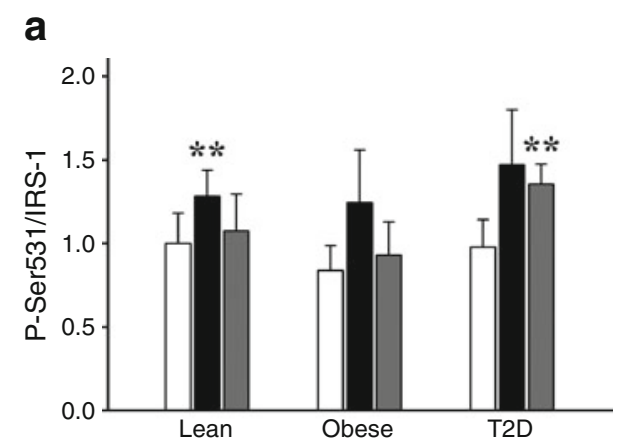

b
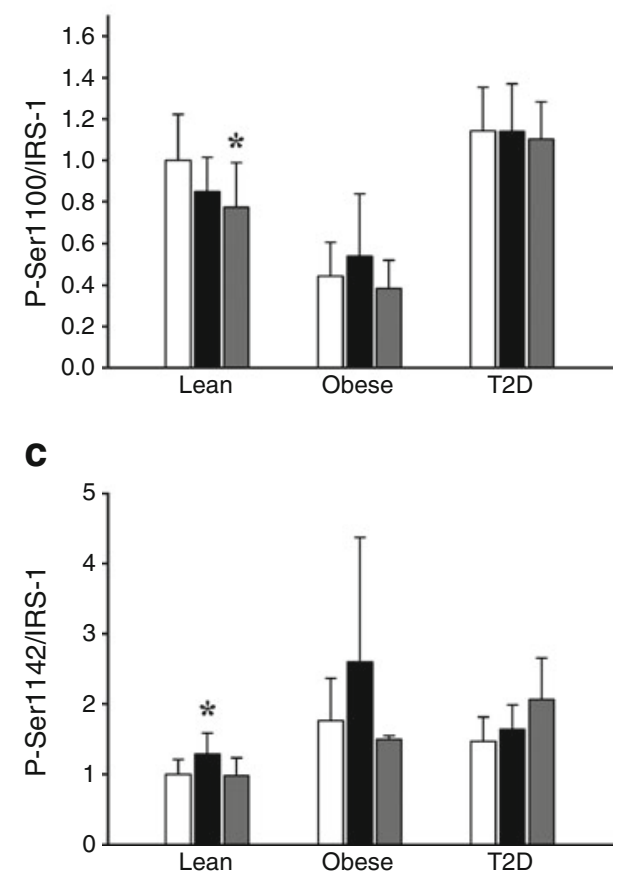

Fig. 3 Insulin-resistant IRS-1 phosphorylation sites in type 2 diabetic muscle in vivo. Experiments were performed identically with those described in Fig. 1. ${ }^{*} p \leq 0.05 ; * * p \leq 0.01$; effect of insulin compared with the $-60 \mathrm{~min}$ time point, within group; $t$ test. T2D, type 2 diabetes 


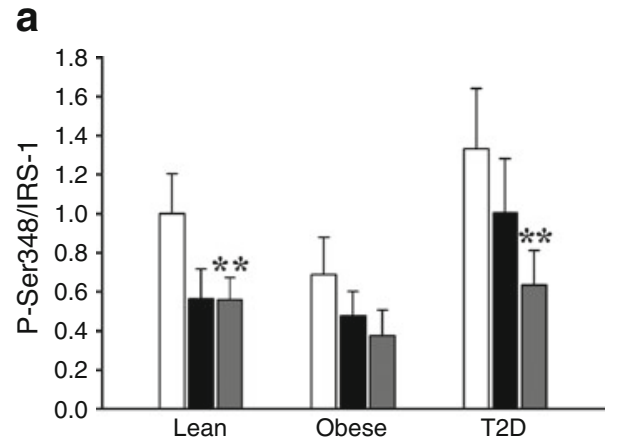

b

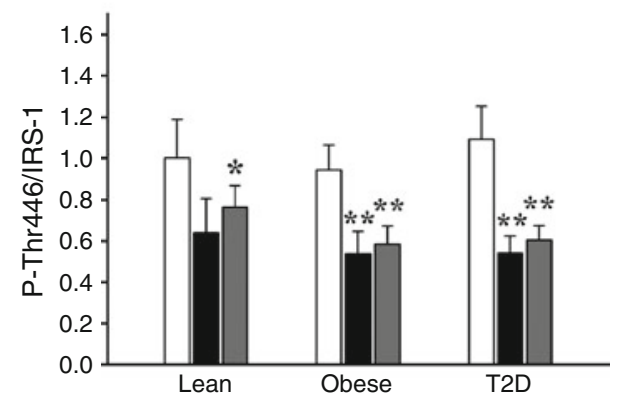

C

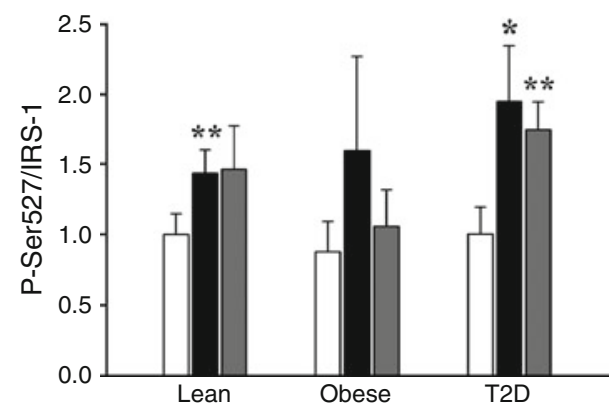

Fig. 4 Insulin-responsive IRS-1 phosphorylation sites in type 2 diabetic muscle in vivo. Experiments were performed identically with those described in Fig. $1 .{ }^{*} p \leq 0.05 ; * * p \leq 0.01$; effect of insulin compared with the -60 min time point, within group; $t$ test. T2D, type 2 diabetes

sites increases upon termination of the insulin signal, acting to interfere with the continued association of IRS-1 with the IR. The increased Ser323 phosphorylation of IRS-1 observed in type 2 diabetes may contribute to insulin resistance by interfering with the insulin-stimulated IR-IRS-1 interaction [44].

Ser636 is a well-documented insulin-stimulated IRS-1 phosphorylation site $[18,45,46]$, and previous in vitro studies have suggested that Ser636 is hyperphosphorylated in models of insulin resistance. In our system, the tryptic peptide that contains Ser629 and Ser636 exists as a mixture of two monophosphorylated, non-coeluting, isobaric phosphopeptides, phosphorylated at either site. Unfortunately, detection of the phosphorylated peptides corresponding to either Ser629 or Ser636 was complicated by an early elution time during HPLC separation. The peaks used for quantification of pSer629 and pSer636 were very broad, and, at times, too broad to acquire a valid peak area value, which may explain the lack of significance observed for the quantification of either of these sites.

Even though our approach allows the simultaneous quantification of a number of phosphorylation sites, some limitations remain. One of the most confounding issues compromising the quantification of IRS-1 phosphorylation in vivo in muscle using this system is the high level of myosin protein located in close proximity to IRS-1 during the SDS-PAGE separation of the IRS-1 immunoprecipitates, as myosin non-specifically immunoprecipitates because of extremely high protein levels. This issue, combined with the low levels of IRS-1 phosphopeptides present in the sample and the heterogeneity between the human participants, may be partially responsible for the inconsistencies observed for quantification values. Another issue is the low fold change that IRS-1 Ser/Thr phosphorylation sites undergo upon insulin stimulation, a phenomenon we have also observed for IRS-1 phosphorylation in vitro, in a cell culture model [23]. We have quantified the phosphorylation of another insulin signalling protein in vitro, raptor, and unlike IRS-1, we observed twofold to fivefold changes in raptor phosphorylation upon insulin stimulation [47], suggesting that the low fold changes in phosphorylation observed for IRS-1 are specific.

One of the more surprising findings was that Thr495 consistently had 40-50\% lower phosphorylation in obese and type 2 diabetic muscle than lean controls, revealing that, in addition to hyper-Ser/Thr phosphorylation of IRS-1, hypophosphorylation may also be involved in compromised IRS-1 function in insulin resistance. This decreased phosphorylation at Thr495 may be due to overactivation of Ser/Thr phosphatases, improper localisation of IRS-1 relative to the Thr495 kinase, or decreased activity of the Thr495 kinase. Thr495 has only been recently identified as an IRS-1 phosphorylation site [22], and, as a result, the kinase responsible for phosphorylating Thr495 and the role of this site in the regulation of IRS-1 function in insulin signal transduction is unknown. In our previous analysis of IRS-1 phosphorylation in muscle, we reported that insulin suppressed Thr495 phosphorylation in lean muscle from four participants. In the current study, the decrease in Thr495 phosphorylation in muscle from lean controls did not achieve significance, although a trend was present. The reason for this difference is not known.

Since the patients with type 2 diabetes were insulin resistant, we predicted that insulin-regulated phosphorylation of IRS-1 observed in the lean controls would be compromised. Paradoxically, however, the insulin-regulated phosphorylation pattern for Ser348, Thr446 and Ser527 
Fig. 5 IRS-1 phosphorylation sites not significantly affected by type 2 diabetes in muscle in vivo. Experiments were performed identically with those described in Fig. 1. T2D, type 2 diabetes a

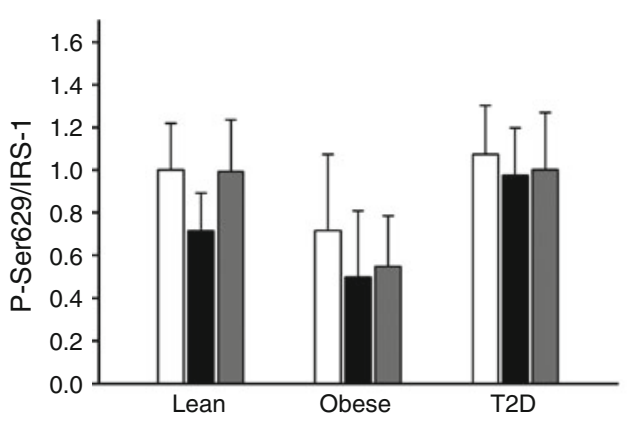

C

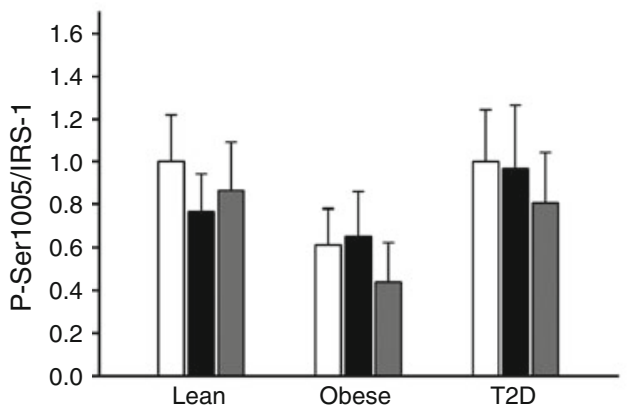

e

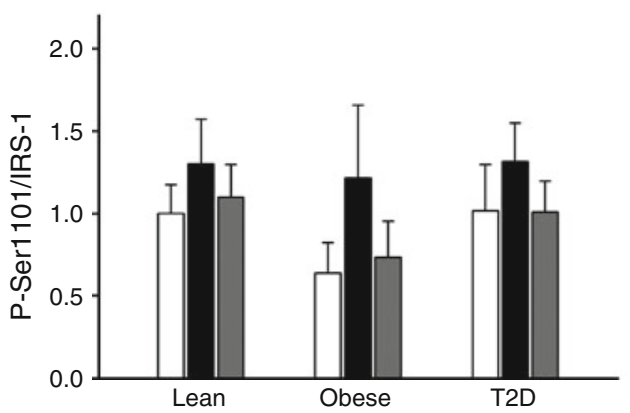

b

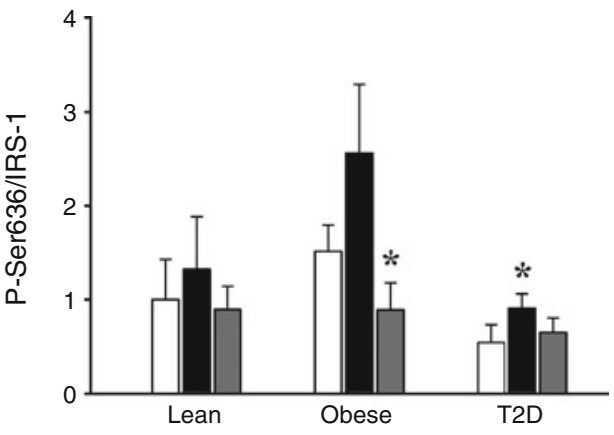

d

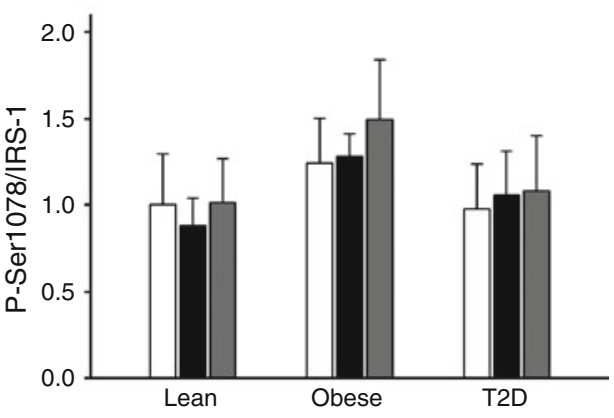

f

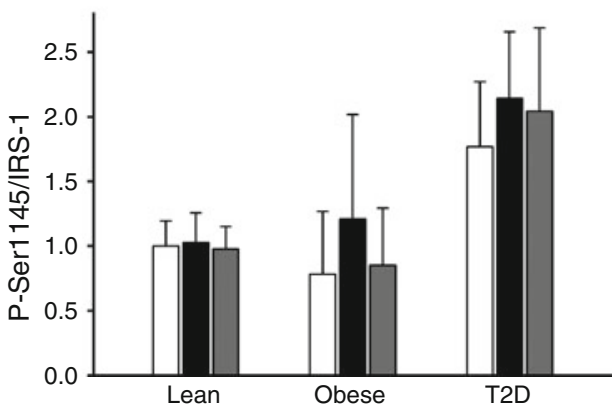

was similar in the type 2 diabetic patients and the lean controls. It is well known that the mitogen activated protein (MAP) kinase pathway is intact or increased in insulinresistant muscle in response to insulin [24], and this pathway may be regulating these IRS-1 phosphorylation sites that behave normally in otherwise insulin-resistant muscle. This may be the case for Ser348 and Thr446, although insulin-stimulated Ser527 phosphorylation has been shown to be under the control of the PI3-K pathway in cultured cells [48]. Insulin-stimulated phosphorylation of IRS-1 at Ser527 (equivalent to Ser522 in the murine/rat sequence) in vitro has previously been reported [48], and our data show that this site undergoes insulin-stimulated phosphorylation in vivo. The remaining IRS-1 phosphorylation sites that were significantly affected by insulin in the lean controls, Ser531, Ser1100 and Ser1142, did not respond significantly to insulin or had a delayed insulin response in the type 2 diabetic participants, indicating that these sites show insulin resistance, implying that there may be dysregulation of kinases or phosphatases for these sites. Little is known regarding IRS-1 phosphorylation at Ser531, Ser1100 and Ser1142, although the insulin-stimulated phosphorylation observed in the lean participants in the present study identifies these sites as novel, previously unreported insulin-regulated IRS-1 phosphorylation sites in human muscle. The observation that these sites show insulin resistance in patients who are insulin resistant in vivo suggests that these sites may play an important role in insulin signalling in vivo that has not been identified using cell culture and animal models.

Insulin does not regulate the function of IRS-1 by phosphorylating one or two major sites, but rather controls IRS-1 by stimulating or suppressing a series of phosphorylation sites. The quantification of IRS-1 by MS allows the simultaneous analysis of multiple phosphorylation sites (16 in this case), providing a more comprehensive 


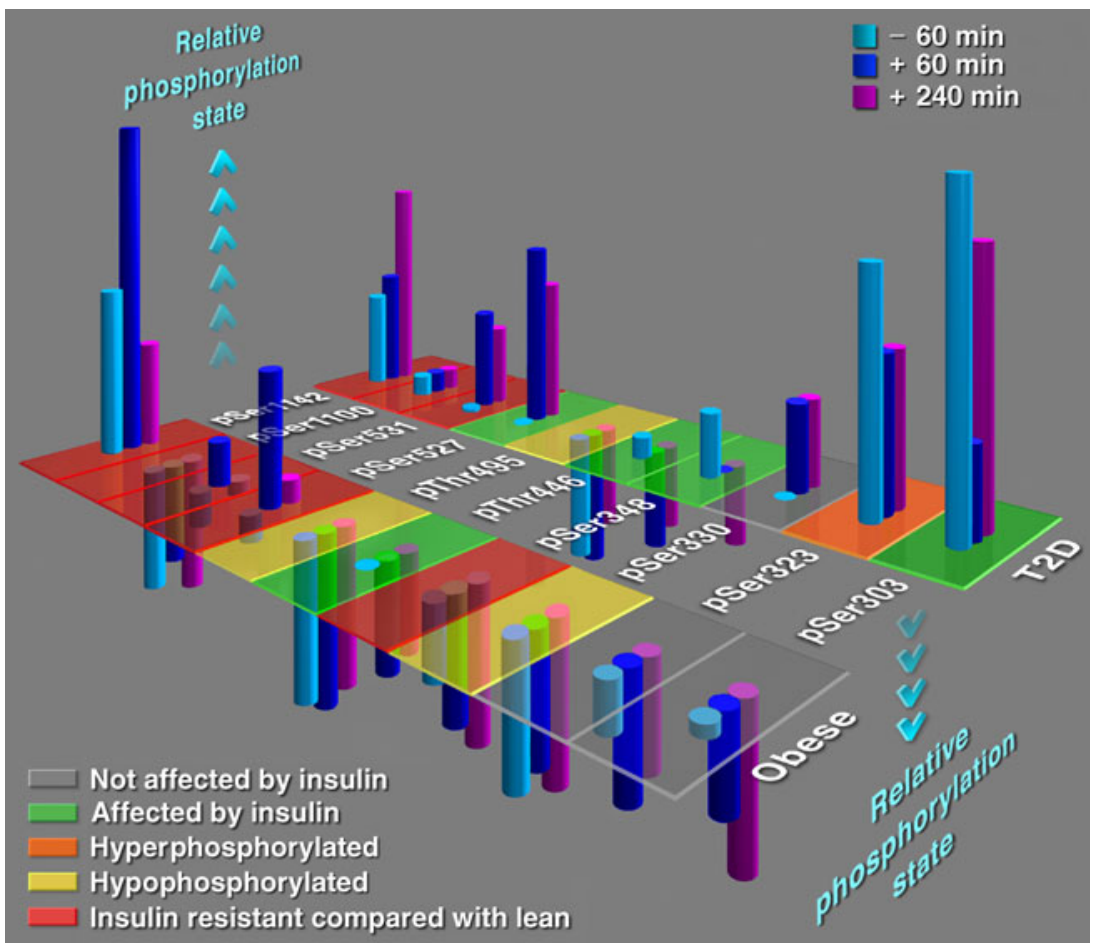

Fig. 6 Summary of IRS-1 phosphorylation. Each IRS-1 phosphorylation site was normalised by the average value of the respective lean basal (-60 min) sample and then expressed as a fold change over the lean basal (identical with Figs 1, 2, 3 and 5). Relative phosphorylation state indicates the status of each phosphorylation site in either obese or type 2 diabetic compared with lean basal, with above the plane representing an increase in phosphorylation and below the plane representing a decrease. The three different coloured cylinders represent

understanding of the overall phosphorylation profile of IRS-1 in insulin action. Although this approach allows insight for the beginning of an understanding of comprehensive, global phosphorylation changes of IRS-1 in type 2 diabetes in vivo in human muscle, a complete picture is still lacking. Future work will aim to obtain complete coverage of IRS-1 by using a combination of different cleavage enzymes, improved MS instrumentation, and incorporation of MS4-based fragment ion quantification. These improvements would increase the number of IRS-1 phosphorylation sites quantifiable by this method and ultimately yield the complete IRS-1 phosphorylation profile. Only after this is achieved can we truly understand the functional relevance of the different phosphorylation patterns of IRS-1 in type 2 diabetes.

In summary, the simultaneous characterisation of the effect of insulin on 16 IRS-1 Ser/Thr phosphorylation sites has resulted in the most comprehensive view of in vivo human muscle IRS-1 phosphorylation to date. We observed both hyper- and hypo-Ser/Thr phosphorylation of IRS-1 in type 2 diabetic muscle. Results from these studies shed new light on how IRS-1 is regulated by Ser/Thr phosphorylation, and how the pattern of IRS-1 the biopsy times, and the five different tiles indicate the notable finding at each phosphorylation site in either obese participants or type 2 diabetics compared with the lean controls. For example, Ser323 was significantly hyperphosphorylated in type 2 diabetes, indicated by the orange tile, whereas Thr495 was significantly hypophosphorylated in type 2 diabetes (yellow tile), while the significant effect of insulin was either preserved (Ser348, Thr446 and Ser527; green tile) or either blocked or delayed (Ser531, Ser1100 and Ser1142; red tile) in type 2 diabetes

phosphorylation becomes distorted in type 2 diabetes. Future studies will aim to understand potential negative feedback mechanisms involving IRS-1 Ser/Thr phosphorylation upon the termination of insulin action in vivo and how these mechanisms might possibly be altered in type 2 diabetic muscle.

Acknowledgements This work was supported in part by NIH grants R01DK47936 (L.J. Mandarino), R01DK66483 (L.J. Mandarino), R01DK081750 (Z. Yi), 5F32DK078460-02 (P. Langlais) and a VA Merit Review grant (C. Meyer) and by the Office of Research and Development, Medical Research Service, Department of Veterans Affairs. We would like to thank Marv Ruona for his help in preparing the summary figure for this article.

Contribution statement PL designed the experiments, processed all the samples, analysed and prepared all the data, and wrote the manuscript. ZY was critical for the design of the MS-based quantification of IRS-1 phosphorylation approach, played an important part in performing the MS, and assisted in writing the technical details of the MS. JF, ML, RM, and PT assisted PL in performing portions of the experiments, contributed to the interpretation of the data and revised the article. EDeF, EP, CM and MM provided oversight of all medical aspects of the study, contributed to the interpretation of the data and revised the article. LJM was actively involved in designing the experiments and drafting the article. All authors approved the final version of the article. 
Duality of interest The authors declare that there is no duality of interest associated with this manuscript.

\section{References}

1. Gual P, Le Marchand-Brustel Y, Tanti JF (2005) Positive and negative regulation of insulin signaling through IRS-1 phosphorylation. Biochimie 87:99-109

2. Zick Y (2003) Role of Ser/Thr kinases in the uncoupling of insulin signaling. Int J Obes Relat Metab Disord 27(Suppl 3):S56S60

3. Haruta T, Uno T, Kawahara J et al (2000) A rapamycin-sensitive pathway down-regulates insulin signaling via phosphorylation and proteasomal degradation of insulin receptor substrate-1. Mol Endocrinol 14:783-794

4. Takano A, Usui I, Haruta T et al (2001) Mammalian target of rapamycin pathway regulates insulin signaling via subcellular redistribution of insulin receptor substrate 1 and integrates nutritional signals and metabolic signals of insulin. Mol Cell Biol 21:5050-5062

5. Sun XJ, Goldberg JL, Qiao LY, Mitchell JJ (1999) Insulin-induced insulin receptor substrate-1 degradation is mediated by the proteasome degradation pathway. Diabetes 48:1359-1364

6. Pederson TM, Kramer DL, Rondinone CM (2001) Serine/ threonine phosphorylation of IRS-1 triggers its degradation: possible regulation by tyrosine phosphorylation. Diabetes 50:2431

7. Shah OJ, Wang Z, Hunter T (2004) Inappropriate activation of the $\mathrm{TSC} / \mathrm{Rheb} / \mathrm{mTOR} / \mathrm{S} 6 \mathrm{~K}$ cassette induces IRS1/2 depletion, insulin resistance, and cell survival deficiencies. Curr Biol 14:1650-1656

8. Paz K, Hemi R, LeRoith D et al (1997) A molecular basis for insulin resistance. Elevated serine/threonine phosphorylation of IRS-1 and IRS-2 inhibits their binding to the juxtamembrane region of the insulin receptor and impairs their ability to undergo insulin-induced tyrosine phosphorylation. J Biol Chem 272:29911-29918

9. Liu YF, Paz K, Herschkovitz A et al (2001) Insulin stimulates PKCzeta -mediated phosphorylation of insulin receptor substrate-1 (IRS-1). A self-attenuated mechanism to negatively regulate the function of IRS proteins. J Biol Chem 276:14459-14465

10. Ravichandran LV, Esposito DL, Chen J, Quon MJ (2001) Protein kinase C-zeta phosphorylates insulin receptor substrate-1 and impairs its ability to activate phosphatidylinositol 3-kinase in response to insulin. J Biol Chem 276:3543-3549

11. Moeschel K, Beck A, Weigert C et al (2004) Protein kinase Czeta-induced phosphorylation of Ser318 in insulin receptor substrate-1 (IRS-1) attenuates the interaction with the insulin receptor and the tyrosine phosphorylation of IRS-1. J Biol Chem 279:25157-25163

12. Aguirre V, Uchida T, Yenush L, Davis R, White MF (2000) The c-Jun $\mathrm{NH}(2)$-terminal kinase promotes insulin resistance during association with insulin receptor substrate-1 and phosphorylation of Ser(307). J Biol Chem 275:9047-9054

13. Liu YF, Herschkovitz A, Boura-Halfon S et al (2004) Serine phosphorylation proximal to its phosphotyrosine binding domain inhibits insulin receptor substrate 1 function and promotes insulin resistance. Mol Cell Biol 24:9668-9681

14. Aguirre V, Werner ED, Giraud J, Lee YH, Shoelson SE, White MF (2002) Phosphorylation of Ser307 in insulin receptor substrate-1 blocks interactions with the insulin receptor and inhibits insulin action. J Biol Chem 277:1531-1537

15. Mothe I, Van Obberghen E (1996) Phosphorylation of insulin receptor substrate-1 on multiple serine residues, 612, 632, 662, and 731, modulates insulin action. J Biol Chem 271:11222-11227
16. Li J, DeFea K, Roth RA (1999) Modulation of insulin receptor substrate-1 tyrosine phosphorylation by an Akt/phosphatidylinositol 3-kinase pathway. J Biol Chem 274:9351-9356

17. Paz K, Liu YF, Shorer H et al (1999) Phosphorylation of insulin receptor substrate-1 (IRS-1) by protein kinase B positively regulates IRS-1 function. J Biol Chem 274:28816-28822

18. Gual P, Gremeaux T, Gonzalez T, Le Marchand-Brustel Y, Tanti JF (2003) MAP kinases and mTOR mediate insulin-induced phosphorylation of insulin receptor substrate-1 on serine residues 307 , 612 and 632. Diabetologia 46:1532-1542

19. Rui L, Aguirre V, Kim JK et al (2001) Insulin/IGF-1 and TNFalpha stimulate phosphorylation of IRS-1 at inhibitory Ser307 via distinct pathways. J Clin Invest 107:181-189

20. Copps KD, Hancer NJ, Opare-Ado L, Qiu W, Walsh C, White MF (2010) Irs1 serine 307 promotes insulin sensitivity in mice. Cell Metab 11:84-92

21. Werner ED, Lee J, Hansen L, Yuan M, Shoelson SE (2004) Insulin resistance due to phosphorylation of insulin receptor substrate-1 at serine 302. J Biol Chem 279:35298-35305

22. Yi Z, Langlais P, De Filippis EA et al (2007) Global assessment of regulation of phosphorylation of insulin receptor substrate-1 by insulin in vivo in human muscle. Diabetes 56:1508-1516

23. Langlais P, Mandarino LJ, Yi Z (2010) Label-free relative quantification of co-eluting isobaric phosphopeptides of insulin receptor substrate-1 by HPLC-ESI-MS/MS. J Am Soc Mass Spectrom 21:1490-1499

24. Cusi K, Maezono K, Osman A et al (2000) Insulin resistance differentially affects the PI 3-kinase- and MAP kinase-mediated signaling in human muscle. J Clin Invest 105:311-320

25. DeFronzo RA, Tobin JD, Andres R (1979) Glucose clamp technique: a method for quantifying insulin secretion and resistance. Am J Physiol 237:E214-E223

26. Lefort N, Glancy B, Bowen B et al (2010) Increased reactive oxygen species production and lower abundance of complex I subunits and carnitine palmitoyltransferase $1 \mathrm{~B}$ protein despite normal mitochondrial respiration in insulin-resistant human skeletal muscle. Diabetes 59:2444-2452

27. Sriwijitkamol A, Christ-Roberts C, Berria R et al (2006) Reduced skeletal muscle inhibitor of kappaB beta content is associated with insulin resistance in subjects with type 2 diabetes: reversal by exercise training. Diabetes 55:760-767

28. Debodo RC, Steele R, Altszuler N, Dunn A, Bishop JS (1963) On the hormonal regulation of carbohydrate metabolism; studies with C14 glucose. Recent Prog Horm Res 19:445-488

29. Lowry OH, Rosebrough NJ, Farr AL, Randall RJ (1951) Protein measurement with the Folin phenol reagent. J Biol Chem 193:265-275

30. Griffin ME, Marcucci MJ, Cline GW et al (1999) Free fatty acidinduced insulin resistance is associated with activation of protein kinase $\mathrm{C}$ theta and alterations in the insulin signaling cascade. Diabetes 48:1270-1274

31. Lee YH, Giraud J, Davis RJ, White MF (2003) c-Jun N-terminal kinase (JNK) mediates feedback inhibition of the insulin signaling cascade. J Biol Chem 278:2896-2902

32. Hirosumi J, Tuncman G, Chang L et al (2002) A central role for JNK in obesity and insulin resistance. Nature 420:333-336

33. Gao Z, Zhang X, Zuberi A et al (2004) Inhibition of insulin sensitivity by free fatty acids requires activation of multiple serine kinases in 3T3-L1 adipocytes. Mol Endocrinol 18:2024-2034

34. Nguyen MT, Satoh H, Favelyukis S et al (2005) JNK and tumor necrosis factor-alpha mediate free fatty acid-induced insulin resistance in 3T3-L1 adipocytes. J Biol Chem 280:3536135371

35. Yuan M, Konstantopoulos N, Lee J et al (2001) Reversal of obesity- and diet-induced insulin resistance with salicylates or targeted disruption of Ikkbeta. Science 293:1673-1677 
36. Perseghin G, Petersen K, Shulman GI (2003) Cellular mechanism of insulin resistance: potential links with inflammation. Int J Obes Relat Metab Disord 27(Suppl 3):S6-S11

37. Gao Z, Hwang D, Bataille F et al (2002) Serine phosphorylation of insulin receptor substrate 1 by inhibitor kappa B kinase complex. J Biol Chem 277:48115-48121

38. Um SH, Frigerio F, Watanabe M et al (2004) Absence of S6K1 protects against age- and diet-induced obesity while enhancing insulin sensitivity. Nature 431:200-205

39. Pende M, Kozma SC, Jaquet M et al (2000) Hypoinsulinaemia, glucose intolerance and diminished beta-cell size in S6K1-deficient mice. Nature 408:994-997

40. Tremblay F, Krebs M, Dombrowski L et al (2005) Overactivation of S6 kinase 1 as a cause of human insulin resistance during increased amino acid availability. Diabetes 54:2674-2684

41. Greene MW, Morrice N, Garofalo RS, Roth RA (2004) Modulation of human insulin receptor substrate-1 tyrosine phosphorylation by protein kinase Cdelta. Biochem J 378:105-116

42. Weigert C, Hennige AM, Brischmann $T$ et al (2005) The phosphorylation of Ser318 of insulin receptor substrate 1 is not per se inhibitory in skeletal muscle cells but is necessary to trigger the attenuation of the insulin-stimulated signal. J Biol Chem 280:37393-37399

43. Weigert C, Kron M, Kalbacher $\mathrm{H}$ et al (2008) Interplay and effects of temporal changes in the phosphorylation state of serine-302, -307, and -318 of insulin receptor substrate- 1 on insulin action in skeletal muscle cells. Mol Endocrinol 22:2729-2740

44. Zick Y (2005) Ser/Thr phosphorylation of IRS proteins: a molecular basis for insulin resistance. Sci STKE 2005:pe4

45. Bouzakri K, Roques M, Gual P et al (2003) Reduced activation of phosphatidylinositol-3 kinase and increased serine 636 phosphorylation of insulin receptor substrate-1 in primary culture of skeletal muscle cells from patients with type 2 diabetes. Diabetes 52:13191325

46. Tzatsos A, Kandror KV (2006) Nutrients suppress phosphatidylinositol 3-kinase/Akt signaling via raptor-dependent mTORmediated insulin receptor substrate 1 phosphorylation. Mol Cell Biol 26:63-76

47. Langlais P, Yi Z, Mandarino LJ (2011) The identification of raptor as a substrate for p44/42 MAPK. Endocrinology 152:1264-1273

48. Giraud J, Haas M, Feener EP et al (2007) Phosphorylation of Irs1 at SER-522 inhibits insulin signaling. Mol Endocrinol 21:2294-2302 